\title{
Addendum
}

\section{Satellite Session : Tropical resources for food animals: new concepts food value, databases, tables and software}

\author{
Maryline Boval
}

\section{Introduction}

As part of SAPT, a satellite chaired by Daniel Sauvant (AFZ, French Association for Animal Production) and Philippe LECOMTE (CIRAD) was held on November 18 focusing on Tropical resources for food animals: new concepts food value, databases, tables and software. This satellite brought together people from 14 different geographic locations, involved in the promotion of tropical feed resources for animal feed in the tropics. Following the presentation of various concepts synthesized in the enclosed pdf, the participants exchanged their views on i) concepts of feed value or multicriteria evaluation of resources ii) evaluation of the available information sources and the methods allowing a better promotion of these resources iii) presentation of the existing databases and studies in progress and iv) their intention to share their data through collaborative projects to be launched in the near future. The following papers present the discussions that were held during this satellite.

\section{Feed value concept and evolution, multi-criteria evaluation}

\author{
Harry Archimède ${ }^{1}$, Denis Bastianelli ${ }^{2}$ and Daniel Sauvant ${ }^{3}$ \\ ${ }^{1}$ INRA, UR143 Unité de Recherches Zootechniques, 97170 Petit-Bourg, France, ${ }^{2}$ CIRAD, Systèmes d'élevage et produits animaux, 34398 Montpellier Cedex 05, \\ France, ${ }^{3}$ AgroParisTech-INRA, UMR 791 MoSAR, 75231 Paris Cedex 05, France \\ Email: Harry.Archimede@antilles.inra.fr
}

Introduction: classic concepts in nutritive and feeding values

Feed evaluation and the animal nutrient requirements were until now the basic concepts underlying animal feeding systems.

Nutritive and feed values are the two approaches for feed evaluation. The Nutritive value (crude protein, soluble and non soluble carbohydrate, minerals...) and their digestibility are measured in vitro or in vivo. The most important components of nutritive value are expressed through feed unit systems. They allow us to estimate the animal nutrient requirements and to express the animal production potential. The Feeding value also takes into account the feed intake. Feeding value could be approximated as intake times digestibility. The nutritive value of a feed resource may vary depending on the other ingredients in the diets. This is the results of positive or negative digestive interactions. The biological laws governing these interactions, particularly in ruminants, have to be known more precisely.
Knowledge on the values of the feed resources

The list of resources of interest varies according to livestock production system. Some tropical feed resources, like cereal (corn, sorgho...) and some protein-rich feeds such as soybeans, peanuts.. are well known because they are the basis of feeding in modern livestock throughout the world. Nevertheless, there are some lesser-known varieties or by-products that could provide variability in the feeding value. A wider variety of resources from tropical biodiversity are still to be characterized accurately. Resources that are not involved in international trade and/or modern agriculture (particularly forages...) are less well known.

The new challenges of animal chains and production systems

It appears urgent to better take into account the new challenges which appeared during the last decades in livestock chains throughout the world. The concrete outcomes of these challenges are subsequent constraints linked to efficiency of 
resource transformation, quality of products, environmental impact of livestock production and animal heath and welfare. Obviously, the classical approach to feeding animals "to meet their requirements and express their potential" is unable to tackle these new challenges. Therefore, this new situation needs a careful re-consideration of the classical feed unit systems (energy, protein...) and the ways to apply them.

\section{The concept of multiple responses to diets}

To take into consideration these new challenges, it is necessary to change our viewpoint in the sense that the target is now to add value to feed resources while optimizing the compromise between the above cited constraints. This new concept can be applied in practice if multiple responses (production, quality of products, feed efficiency, emissions on environment, animal health and welfare) of the animals to the diets or feeding practices are known and modelled. As a consequence, this new multicriteria evaluation system requires the following: 1 ) identifying the criteria that are to be optimized; 2 ) modelling the marginal responses of each criteria to the diets and dietary practices; 3) identifying the inherent relationships among the responses to each determinant, providing information on the possibilities of compromise between antagonistic responses. For example the fibre content of the diets of sows is now increasing to improve their well being status, however this choice decreases the dietary energy and thus the animal growth. Nitrogen concentration of feed is also an example that illustrates some antagonism that can exist between maximizing animal performance and reducing nitrogen emission via faeces and particularly urine; 4) providing the weights for criteria according to the importance accorded to them, even economical weights; 5) Defining tools to achieve these optimizations.

Consequences in building feed databases:

In turn, it is necessary to try to shift the concept of 'nutritive value' of feed (concentration in various key nutrients or feed units) to a new concept of 'response potential' of feed. For this, each feed would be characterized in terms of its contribution to response (i.e. environmental value....). It must be stressed that some classical components of nutritive value are also suited to predict responses. For instance OM digestibility is a key item to predict both nutritive (energy) value and feed efficiency. A major limit of characterizing the feed by the 'potential of responses' is that it assumes a basic principle of additivity. For instance, as global warming is becoming a major issue, classification of feeds based on their potential to produce methane could be a priori interesting. However, interactions between feed within diets are so marked that $\mathrm{CH} 4$ production cannot be a feed attribute. In contrast, it is a dietary characteristic.

New potential of responses of feed can be defined. For example, in warm areas, the ability of the feed to allow the animal to better fight against the heat would be an important criterion. This would indicate that it is necessary to differentiate between feeds on their ability to induce extra heat, on their capacity to buffer the water balance and minerals related to sweating.

The multicriteria approach should also incorporate the concept of risk to the animal, for instance under intensive conditions, ruminants face the risk of acidosis and some feed characteristics can allow reducing the risk of acidosis. Otherwise, risk for the whole food chain could also be considered for feed containing undesirable anti nutritive factors.

\section{Modeling multiple responses}

The multricriteria approach will take knowledge or information from systemic modelling. This allows us to integrate the endogenous and exogenous controls of metabolic flux which determine responses to the diet. Moreover systemic modelling presents animal responses under different context. The new concept of Feed multi criteria evaluation has to be included in the larger approach of farming system which is also evaluated for multiple functions.

\title{
Feed and diet value evaluation from literature data and from the existing databases
}

\author{
Daniel Sauvant ${ }^{1,2}$, Gilles Tran $^{2}$, Valérie Heuze ${ }^{2}$, Denis Bastianelli ${ }^{3}$ and Harry Archimede ${ }^{4}$ \\ ${ }^{1}$ AgroParisTech-INRA, UMR 791 MoSAR, 75231 Paris Cedex 05, France; ${ }^{2}$ Association Française de Zootechnie, 75231 Paris Cedex 05, France; ${ }^{3}$ CIRAD, Systèmes \\ d'élevage et produits animaux, 34398 Montpellier Cedex 05, France; ${ }^{4}$ INRA, UR143 Unité de Recherches Zootechniques, 97170 Petit-Bourg, France \\ Email: sauvant@agroparistech.fr
}

The production of information on feed characteristics is increasing rapidly and numerous data bases are now built in various organizations (research, universities, private companies...). From all these data, several methods can be applied to predict nutritive and feeding values of resources.
These methods are more or less easy to apply, accurate, repetable, costly, and there is a concern on the way to combine all this heterogenous information into a consistent frame to obtain ultimate reference values. The present feed tables, which were built through various ways, can differ 